Dort wird auch der Nationalatlas bearbeitet. In der Exkursionsvorbereitung wird vom Exkursionsleiter ein Begleitmaterial zu den historischen Karten des Iran begonnen, mit den frühesten arabischen Karten über die Weitere Informationen sind auf

\section{DGfK-Mitgliederversammlung 2013 Einladung}

Hiermit lade ich alle Mitglieder der Deutschen Gesellschaft für Kartographie e.V. (DGfK) zur diesjährigen Mitgliederversammlung ein. Sie findet anlässlich des 61. Deutschen Kartographentages in Dresden statt am Mittwoch, dem 28. August 2013, von 14:00 Uhr bis 17:00 Uhr, im Saal des "Blockhauses”, Große Meißner Straße 19, 01097 Dresden. Als vorläufige Tagesordnung schlägt der Vorstand vor:

1. Begrüßung und Feststellung der ordnungsgemäßen Einladung und der Beschlussfähigkeit

2. Ehrung der verstorbenen Mitglieder

3. Genehmigung der Tagesordnung

4. Genehmigung des Protokolls der letzten Mitgliederversammlung (veröffentlicht in KN 6/2012, S. 341-343)

5. Ehrung verdienter Mitglieder

6. Bericht des Präsidenten

7. Bericht des Schatzmeisters

8. Bericht des Sekretärs

9. Bericht des Hauptschriftleiters der KN

10. Bericht der Kassenprüfer

11. Aussprache zu den Punkten 6-10

12. Antrag auf Entlastung des Vorstandes

13. Bericht des Geschäftsführers der Geomatik-Tagungs-GmbH

14. Vorstandswahl - Bekanntgabe des Wahlergebnisses

15. Genehmigung des Haushaltsvoranschlages 2014

16. 62. Deutscher Kartographentag 2014 in Hamburg

17. 63. Deutscher Kartographentag 2015 in Stuttgart

18. Änderung der Satzung; hier: Weiterentwicklung der DGfK - Einführung eines Namenszusatzes

Gemäß § 15 Abs.1 der Satzung beantragt der Vorstand der DGfK, den Namen des Vereins „Deutsche Gesellschaft für Kartographie e.V. (DGfK)" zu ändern in "Deutsche Gesellschaft für Kartographie e.V. (DGfK) - Gesellschaft für Kartographie und Geomatik". Begründung: Mit dem Namenszusatz wird auf Anregung zahlreicher Mitglieder der Tatsache Rechnung getragen, dass das Ausbildungsberufsbild "Kartograph/in" zu Gunsten des Ausbildungsberufsbildes "Geomatiker/in" aufgegeben und Studiengänge an mehreren Hochschulen unter Verwendung der Bezeichnung "Geomatik" neu ausgerichtet wurden. Die DGfK möchte mit der Namenserweiterung unterstreichen, dass sie sich selbstverständlich als Verband auch der neuen Berufe versteht.

19. Berichte aus den Sektionen und Kommissionen

20. Verschiedenes

Stellungnahmen oder weitere Vorschläge zur Tagesordnung richten Sie bitte bis zum 20. August 2013 an den Sekretär (sekretaer@dgfk.net).

Prof. Dr. Manfred Weisensee

Präsident der DGfK unserer Internetseite unter http:// halle-leipzig.dgfk.net zu finden. Für Voranmeldungen und Anfragen wenden Sie sich bitte direkt an Professor Max Linke unter Tel. 03443/202674 oder unter E-mail olinke@t-online.de.

Thomas Chudy

Sektion Halle-Leipzig

\section{Ravenstein-Förderpreis 2013}

\section{Aufruf zur Teilnahme}

Alljährlich wird im Sinne der Stifterin, Frau Helga Ravenstein †, der Ravenstein-Förderpreis durch die Kartographie-Stiftung Ravenstein in enger und partnerschaftlicher Zusammenarbeit mit der DGFK verliehen. Ziel der Stiftung und des Preises ist die Nachwuchsförderung der deutschen Kartographie und der damit verbundenen Ausbildungsberufe und Studienrichtungen der Geoinformationstechnologie. Teilnahmeberechtigt am Wettbewerb sind Auszubildende der Berufe der Geoinformationstechnologie sowie alle Studierenden der Geowissenschaften und deren Nachbardisziplinen, die mit Ihrer Arbeit die Leistungsfähigkeit des Nachwuchses für einen weiteren beruflichen Erfolg im Bereich der Geoinformationstechnologie demonstrieren.

Der Ravenstein-Förderpreis besteht aus einem Preisgeld und einer Urkunde. Alle Teilnehmer sowie die Ausbildungsbetriebe erhalten eine Teilnahmebestätigung. Die Preisträger werden zur Preisverleihung am 24. September 2013 in das Bundesamt für Kartographie und Geodäsie nach Frankfurt/Main eingeladen.

Das Preisgeld wird in Teilpreisen für die beste Arbeit von Studierenden der Geowissenschaften und von Auszubildenden der Berufe der Geoinformationstechnologie aufgeteilt. Sie werden in der KN Kartographische Nachrichten, der Fachzeitschrift der DGfK, veröffentlicht. Die Kommission Aus- und Weiterbildung der DGfK wird die Preisträger und ihre Arbeiten auf ihrer Internetseite vorstellen: http:// www.kartographie-ausbildung.de

Die Arbeiten werden durch eine unabhängige Jury bewertet, die aus mindestens fünf Personen aus verschiedenen Bereichen der Kartographie besteht. Die Entscheidungen der Jury sind nicht anfechtbar. Sollten unter den eingereichten Arbeiten mehrere gleichwertige vorliegen, kann die Jury eine andere Verteilung des Preisgeldes vornehmen.

Teilnahmebedingungen des Wettbewerbes:

- Die eingereichten Arbeiten müssen eigenständig entwickelte Produkte sein, die aus technischer, gestalterischer oder wissenschaftlicher Sicht ein Berufsfeld der Geoinformationstechnologie repräsentieren.

- Als Formate sind alle Arten von Ausdrucken sowie die für den Druck nötigen Dateien einzureichen, keine Originale!

- Den Arbeiten ist eine Erklärung beizulegen, dass sie selbstständig und ohne fremde Hilfe angefertigt wurden;

- ihr ist ein Quellenverzeichnis beizulegen und eine Bestätigung, dass das Urheberrecht beachtet wurde;

- zum Wettbewerb können Arbeiten von Einzelnen oder von Gruppen (bis zu fünf Mitarbeitern) eingereicht werden;

- die Arbeiten dürfen nicht älter als zwei Jahre sein;

- den Arbeiten ist eine Kurzbeschreibung (max. 2 Seiten DIN A 4) beizufügen, die Auskunft über Zweck, Zielgruppe und Intention, Wahl der Darstellungsmittel und Herstellungsverfahren gibt. Bei rechnergestützt hergestellten Arbeiten sind die technischen Voraussetzungen anzugeben;

- die Arbeiten müssen auf der Rückseite mit Namen, Anschrift und E-Mail-Adresse der Teilnehmer sowie der Ausbildungsstätte oder der Hochschule versehen sein;

- den Arbeiten ist ein adressierter Aufkleber für die Rücksendung beizulegen. 